\title{
Tract Size in Percutaneous Nephrolithotomy (PCNL) : Does It Really Matter?
}

\author{
Mofizur Rahman ${ }^{1 *}$ \\ Mohammed Monowar-Ul-Hoque ${ }^{1}$ \\ Kazi Md Monwarul Karim ${ }^{1}$ \\ A K M Akramul Bari ${ }^{1}$ \\ Tanvir Rahman ${ }^{1}$ \\ Asaduzzaman Asad ${ }^{1}$
}

'Department of Urology

Chattogram Medical College

Chattogram, Bangladesh.

*Correspondence to:

Dr. Mofizur Rahman

Assistant Professor

Department of Urology

Chattogram Medical College

Chattogram, Bangladesh.

Mobile : +8801823050720

Email: euromofiz@yahoo.com

Date of Submission : 12.05 .2019

Date of Acceptance : $\quad 30.06 .2019$

www.banglajol.info/index.php/CMOSHMCJ

\begin{abstract}
Background : Percutaneous Nephrolithotomy (PCNL) is the treatment of choice in removal of renal and proximal ureteral calculi. The primary goal of PCNL is to achieve stone free status while minimizing morbidity and complications. In recent years, the instruments used have been miniaturized in an effort to decrease morbidity associated with standard PCNL as well as increase the efficacy of stone removal. The aim of this study is to compare the safety and efficacy of PCNL using different tract size.
\end{abstract}

Materials and methods: This hospital based prospective interventional study was conducted on patients with 1 to $4 \mathrm{~cm}$ renal stones who underwent PCNL either by Minior Standard PCNL technique in Chattogram Medical College Hospital and different private hospitals in Chittagong from July 2016 to June 2018. Patients aged above 12 years of age, irrespective of gender with normal renal function were evaluated to compare stone clearance, total operative time, need for blood transfusion, postoperative pain and other complications. Those who had previous history of open renal surgery, active urinary tract infection, renal malformation, uncorrected coagulopathy and morbid obesity were excluded.

Results: A total of 64 patients were enrolled consecutively for PCNL who were divided equally into two groups randomly for minimally invasive PCNL (Mini$P C N L$ ) and Standard PCNL. The average stone size in mini-PCNL group was 2.64 $\pm 0.94 \mathrm{~cm}$ and $2.776 \pm 0.97 \mathrm{~cm}$ in standard-PCNL group. Mean tract size was $18.44 \pm 1.32 F(16-20)$ and $26.7 \pm 5 F(24-30)$ respectively. In mini-PCNL operative time was significantly longer than that of standard PCNL with 110.31 \pm 21.77 vs $95.94 \pm 19.82$ min respectively. Conversely, there was an advantage of mini-PCNL over the standard one in terms of a significantly reduced hemoglobin drop $(0.5 \pm 0.25$ vs. $0.8 \pm 0.34)$ gram and hospital stay $(2.13 \pm 0.79$ vs.3.38 \pm 1.13 days) respectively though there was no statistical difference in terms of stone clearance rates between two groups (86.7\% vs. 93.33\%). There was no statistical difference in terms of Visual Analogue Scale (VAS) score (5.44 \pm 1.5 vs.6.19 \pm 1.65$)$ for pain perception. The complication rate of mini-PCNL had no significant difference with that of standard PCNL (10\% vs $13.6 \%$ ). No statistical difference was recorded in terms of postoperative fever $\left(\geq 38^{\circ} \mathrm{C}\right)$ between two groups (2 in each group, 6.67\%, $p=1$ ). Blood transfusion requirement was much less in mini PCNL group (10\% vs. 33.33\%).

Conclusion: In addition to minimal bleeding and excellent stone clearance, miniPCNL has several features for which it should be considered as an alternative or adjunct to standard PCNL, URS and ESWL. These include safe supra-costal puncture, excellent access to nearly all calyces and upper ureter, less hospital stay and suitable for large stones also. Future studies should continue to refine methods to assess complexity and safety and to determine consensus on the use of mini- PCNL. 
Key words: Percutaneous Nephrolithotomy (PCNL); M inimally invasive PCNL (M ini-PCNL); Standard PCNL.

\section{INTRODUCTION}

$\mathrm{K}$ idney stone is the third most common disease of urinary tract. Peak incidence of stone occurrence is the fourth to sixth decades of life. M en are affected two to three times more often than women. Stone formation has migrated from the lower to the upper urinary tract with westernization of global culture and the disease is getting increasingly gender blind ${ }^{1}$. Over the last two decades, Percutaneous Nephrolithotomy (PCNL) and Extracorporeal Shock Wave Lithotripsy (ESWL) have replaced open surgery for the management of kidney stones ${ }^{2}$. With the use of invasive and noninvasive techniques, open surgery has been used only at a rate of $1-2 \%$ in the management of renal stones $^{3}$. PCNL was first described by Fernström and J ohansson in 1976. According to the updated European A ssociation of U rology (EAU) guidelines, PCNL has been recommended as the therapy of choice for large renal calculi $(>20 \mathrm{~mm})$ and also for smaller stones $(10-20 \mathrm{~mm})$ of the lower renal pole when unfavorable factors for ESW L exist. Excellent Stone-Free Rates (SFR) following PCNL have been reported, which range from $76 \%$ to $98 \%{ }^{4}$. However, PCNL is still a challenging surgical technique and can be associated with significant complications that may compromise its efficacy. In order to decrease morbidity associated with larger instruments like blood loss, postoperative pain and potential renal damage, a modification of the technique of standard PCNL has been developed. This is performed with a miniature endoscope via a small percutaneous tract $(11-20 \mathrm{~F})$ and was named as minimally invasive PCNL or mini-PCNL or mini-Perc. This technique was first developed and accomplished by Jackman et al. in the pediatric population with the use of an $11 \mathrm{~F}$ access tract $^{5}$. Since then, the method has become a treatment option for adults as well ${ }^{6}$. The primary goal of PCNL is to achieve stone-free status while minimizing morbidity and complications. Usually, the term mini-PCNL is used for access sheaths $20 \mathrm{Fr}$ or below $20 \mathrm{Fr}$. However, the terminology has not been standardized yet and the procedure lacks a clear definition ${ }^{7}$. A ccess sizes ranging from $11 \mathrm{~F}$ to $20 \mathrm{~F}$ have been reported in the some literature, whereas 14 to $20 \mathrm{~F}$ has been described in some other papers ${ }^{8,9}$. The mini-perc technique is believed to have several advantages, including decreased blood loss, increased maneuverability, less pain and shorter hospital stays. As the risk for bleeding complications is related to the number and caliber of tracts used, limited transfusion rates have been reported with this technique ${ }^{10,11}$. However, reducing tract size may adversely affect some procedure related factors. Although several studies have shown the safety and eficacy of mini-PCNL for small calculi, the fear of reduced visibility, a prolonged operating time, and lower primary stone-free rates owing to the reduced shaft diameter has resulted in reluctance to apply miniaturized instruments in patients with a larger stone burden $^{12}$. Thus, each of the types of PCNL has its own advantages and disadvantages and has been selected by different surgeons. Moreover, the choice also depends on surgeons experience and expertise. In Bangladesh, due to recent advancement of endourology, PCNL is frequently done for retrieval of renal stone and one of the fundamental steps of PCNL is the creation of the nephrostomy access. There is lack of data comparing different techniques in our context. Therefore, it was necessary to conduct further research to evaluate the efficacy and safety of mini-PCNL. Therefore, we considered it is necessary to perform a prospective interventional study in our setting comparing the efficacy and safety of the mini-percutaneous nephrolithotomy in comparison to standard PCNL. The aim of this study was to demonstrate that mini-percutaneous nephrolithotomy might represent a reasonable procedure in patients with smaller stones offering a similar outcome as standard percutaneous nephrolithotomy with advantage of reduced morbidity. Our objectives were to evaluate the efficacy and safety to compare the stone clearance rate to observe the access time, fluoroscopy time for access and total operative time to compare the postoperative pain between two groups, to evaluate any per operative or immediate postoperative complications like excessive bleeding, peri-operative transfusion requirements, post-operative fever and urinary leakage, post-operative hospital stay between two groups.

\section{MATERIALS AND METHODS}

This randomized controlled study was done in the U rology Department, Chattogram Medical College Hospital and different Private Hospitals of Chattogram, Bangladesh from July 2016 to J une 2018, based on patients admitted with renal calculi and underwent PCNL during the study period. Patients was selected by consecutive sampling method considering the inclusion and exclusion criteria \& divided randomly into two groups using an online based statistical calculator (Graph Pad Software, Inc. 2017, https://www.graphpad.com/ quickcalcs /randomizel.cfm). Group A for the miniaturized PCNL technique and Group B for the conventional technique. To estimate the sample size for hypothesis testing, standardstatistical formula was used. Patients underwent PCNL for renal stone, where patients age 12 years or more, stone size $1 \mathrm{~cm}$ to $4 \mathrm{~cm}$, with normal renal function were evaluated for study. Those who had previous history of open renal surgery, active UTI, renal malformation, uncorrected coagulopathy \& morbid obesity were excluded.

After proper counseling and detailed explanation of procedure, obtaining written informed consent, all patients were evaluated by detailed history, thorough physical examinations and relevant investigations. Negative urine culture were ensured before surgical intervention. Co-morbidities were addressed and controlled preoperatively.

All patients underwent PCNL under general anesthesia and received intravenous broad spectrum antibiotics prior to manipulation. Initially, on lithotomy position, a 6 Fr ureteric 
catheter was placed transurethrally over the 0.035 inch guide wire under fluoroscopic guidance. Then patient was placed at prone position. Pelvi-calyceal system of the targeted while kidney was opacified with diluted contrast media under fluoroscopy. Initial puncture was decided on retrograde pyelogram findings. Percutaneous access was created using an $18 \mathrm{G}$ access needle into the selected calyx. A guidewire was placed into the collecting system. The nephrostomy tract was dilated by gradual dilatation technique with Metallic Alkan Dilator (K arl Storz) 16 to $20 \mathrm{Fr}$ for mini PCNL and 24 to $28 \mathrm{Fr}$ for standard PCNL. Then A mplatz sheath (Cook / Rusch Medical) was positioned into the renal collecting system accordingly. The stone was disintegrated using pneumatic lithotripter. Nephroscope with forceps was used to retrieve stones from calyces. Once complete clearance was confirmed fluoroscopically and endoscopically, a 5/6 F J J stent was placed antegradely. On completing the procedure, the A mplatz sheath was removed after keeping a nephrostomy tube in situ.

On postoperative day 1 , a plain X-ray was obtained to document stone clearance. Nephrostomy tube was removed if the urine was not hemorrhagic and stone clearance was achieved. The Foley's catheter was removed on $2^{\text {nd }}$ postoperative day. The JJ stent was removed after 4 weeks. Re-PCNL, URS (Ureterorenoscopy) and ESWL was considered as accessory treatment alternatives when indicated. Close follow up and recording was done to search for any complications encountered immediately after operation. $\mathrm{Hb} \%$ will be estimated on $1^{\text {st }}$ post-operative day. Post-operative pain was measured using "Visceral analogue Score (VAS)". A ny need for blood transfusion was recorded. Patients were discharged with appropriate follow up advices if no complication arises.

\section{RESULTS}

The average stone size in mini-PCNL group was $2.64 \pm 0.94$ $\mathrm{cm} \& 2.776 \pm 0.97 \mathrm{~cm}$ in standard-PCNL group $(p=0.6)$. M ean tract size was $18.44 \pm 1.32 \mathrm{~F}(16-20) \& 26.7 \pm 5 \mathrm{~F}$ (24-30) respectively with $p$ value $<0.001$. In mini-PCNL operative time was significantly longer than that of standard PCNL with $110.31 \pm 21.77$ vs. $95.94 \pm 19.82$ minrespectively with $p=0.007$. Conversely, there was an advantage of mini-PCNL over the standard one in terms of a significantly reduced hemoglobin drop $(0.5 \pm 0.25 \mathrm{gm}$ vs . $0.8 \pm 0.34$ gram $\%$, $p<$ $0.001)$ and less hospital stay $(2.13 \pm 0.79$ vs. $3.38 \pm 1.13$ days, $p<0.001$ ), respectively though there was no statistical difference in terms of stone clearance rates between two groups ( $86.7 \%$ vs. $93.33 \%, p=0.39)$. There was no statistical difference in terms of Visual A nalogue Scale (VAS) score (5.44 \pm 1.5 vs. $6.19 \pm 1.65)$ for pain perception. The complication rate of miniPCNL had no significant difference with that of standard PCN L (10 \% vs. $13.6 \%, p=0.69)$. No statistical difference was recorded in terms of postoperative fever $\left(\geq 38^{\circ} \mathrm{C}\right)$ between two groups ( 2 in each group, $6.67 \%, p=1$ ), blood transfusion requirement was much less in min-PCNL group (10\% vs. $33.33 \%, p=0.02$ was significant).
Table I : Demography of patients in the mini-PCNL \& conventional percutaneous nephrolithotomy groups (Mean \pm SD or \%)

\begin{tabular}{|c|c|c|c|}
\hline & $\begin{array}{l}\text { Mini-PCNL } \\
(n-32)\end{array}$ & $\begin{array}{l}\text { PCNL Conventional } \\
\text { (n-32) }\end{array}$ & p value \\
\hline Age (Year) & $40.34 \pm 12.19$ & $42.34 \pm 13.33$ & 0.4 \\
\hline Sex & Female $38 \%$ & $\mathrm{M}$ ale $72 \%$ Female $28 \%$ & 0.28 \\
\hline
\end{tabular}

Table II : Surgical parameters in the mini-PCNL \& conventional percutaneous nephrolithotomy groups (M ean \pm SD or $\%$ )

\begin{tabular}{lrrr} 
& Mini-PCNL Group A & Standard-PCNL Group B & p value \\
Number of patients & 32 & 32 & \\
Stone size & $2.64 \pm 0.94$ & $2.776 \pm 0.97$ & 0.6 \\
M ean Tract size & $18.44 \pm 1.32$ & $26.7 \pm 5$ & $<0.001$ \\
Operative time & $110.31 \pm 21.77$ & $95.94 \pm 19.82$ & 0.007 \\
Hemoglobin drop & $0.5 \pm 0.25$ & $0.8 \pm 0.34$ & $<0.001$ \\
Hospital stay & $2.13 \pm 0.79$ & $3.38 \pm 1.13$ & $<0.001$ \\
Stone clearance rate & 86.7 & 93.33 & 0.39 \\
Visual analoque scale & $5.44 \pm 1.5$ & $6.19 \pm 1.65$ & $>0.06$ \\
Complication rate & 10 & 13.6 & 0.69 \\
Postoperative fever & 6.67 & 6.67 & 1 \\
Blood transfusion & & & \\
requirement & 10 & 33.33 & 0.02 \\
\hline
\end{tabular}

\section{DISCUSSION}

The "mini-perc" or mini-PCNL technique was first developed for children and reported in $1997^{13,14}$. The advantages of the "mini-perc" technique highlighted by these authors included decreased renal and body wall trauma, adjustable length, and convenient working size for pediatric instruments.

Even in the first series regarding mini-PCNL, the Stone Free Rate (SFR) was high enough although the stone burden was relatively low. Jackman et al. reported an SFR of $85 \%$ in children and $89 \%$ in adults with a stone burden $1.2 \mathrm{~cm}^{2}$ and 1.5 $\mathrm{cm}^{2}$ respectively ${ }^{12}$. Similar SFRs between mini-PCNL and PCNL have been reported by most authors except Giusti et al. who reported lower SFRs despite longer operative times ${ }^{15}$. Similar to the pediatric experience, early stone-free success with the adult "mini-perc" was equivalent to that of recent series. Saxby et al. and L ingeman et al. have reported stone-free rates of $85 \%$ and $95 \%$ respectively for stones measuring $2.0 \mathrm{~cm}$ or less in size $e^{16,17}$. This is consistent with the $86.7 \%$ stone-free rate of the current series. Additionally, in the kidney in which a small residual fragment was seen 1 week postoperatively there is a reasonable chance of spontaneous passage.

Jackman SV has mentioned limited blood loss, increased maneuverability, decreased postoperative pain \& limited hospital stay in cases of mini-PCNL ${ }^{11}$. Limitations of the procedure include the necessity to disintegrate stones into small enough fragments to fit through a reduced sizedsheath which results in longer operative times. 
Cheng et al. stated no significant differences in SFR between the mini-PCNL and PCNL ${ }^{18}$. However, a better stone clearance rate was demonstrated for multiple calyceal stones when miniPCNL was performed (85.2\% vs. 70\%). Zhong W et al. stated Higher SFR was achieved in the treatment of staghorn stones with mini-PCNL and the creation of multiple access tracts $(89.7 \% \text { vs. } 68 \%)^{19}$.

Giusti et al. and M ishra et al. has found longer operative times associated with mini-PCNL (155.5 min and $45 \mathrm{~min}$ vs. 106.6 $\min$ and 31 min respectively) ${ }^{15,20}$. A bdelhafez et al. stated $M$ ini-PCNL as more effective when treating smaller $(<20 \mathrm{~mm})$ rather than larger $(>20 \mathrm{~mm}$ ) renal stones (SFR $90.8 \%$ vs. $76.3 \%)^{21}$. In current series operating time $110.31 \pm 21.77$ were significantly larger than standard PCNL, which is very similar with those previous study.

Traxer et al. measured and compared the extent of renal parenchyma injury also concluded that renal parenchyma damage resulting from the creation of a nephrostomy tract is small compared to overall renal volume regardless of the size of the nephrostomy tract ${ }^{22}$. However, the benefit of mini-PCNL remains as the use of smaller access sheaths resulted in reduced intraoperative blood loss, less postoperative pain and shorter hospital stay. Cheng et al. and $\mathrm{M}$ ishra et al. stated an advantage of mini-PCNL over the conventional procedurein terms of a significantly reduced hemoglobin drop $(0.53 \mathrm{~g} / \mathrm{dl}$ and $0.8 \mathrm{~g} / \mathrm{dl}$ vs. $0.97 \mathrm{~g} / \mathrm{dl}$ and $1.3 \mathrm{~g} / \mathrm{dl}$ respectively) and the need for blood transfusion ( $1.4 \%$ vs. $10.4 \%)^{18,20}$. M ishra et al. concluded that analgesic requirement has also been found significantly decreased in mini-PCNL when compared to standard PCNL (55.4 g vs. $70.2 \mathrm{~g}$ tramadol $)^{20}$. Knoll et al. and $\mathrm{M}$ ishra et al. stated that hospital stay was significantly shorter after mini-PCNL (3.8 days and 3.2 days vs. 6.9 days and 4.8 days respectively $)^{23,20}$. In our series Hospital stay was also significantly shorter after mini-PCNL $(2.13 \pm 0.79 \vee 3.38$ \pm 1.13 days) less post operative pain were also noted (Visual analogue scale: $5.44 \pm 1.5 \vee 6.19 \pm 1.65$ ) diminished $\mathrm{Hb} \%$ drops $(0.5 \pm 0.25 \vee 0.8 \pm 0.34)$ less blood transfusion $(10 \% \vee$ $33.33 \%)$.

\section{CONCLUSION}

The technique and rational for performing a "mini-PCNL" in selected patients are presented in this report. Early experience suggests that its stone clearance rate is comparable with that of a standard PCNL and that it may have advantages over the current procedure. These potential advantages include decreases in blood loss, transfusion rate, pain, and length of hospital stay. More extensive studies are needed to validate these hypotheses. It is likely that this technique will become an important alternative in the endourologist's armamentarium for the quick and definitive treatment of smaller stones in selected patients.

\section{DISCLOSURE}

A Il the authors declared no competing interest. 


\section{REFERENCES}

1. Pearle M S, A ntonelli JA, L otan Y. U rinary Lithiasis: Etiology, Epidemiology, and Pathogenesis. Wein A J, Kavoussi, LR. Partin, AW \& Peters CA, (eds), Campbell-Walsh U rology, $11^{\text {th }}$ edn. 2016;1188-1199. Elsevier, Philadel phia.

2. Segura JW, Preminger GM, A ssimos DG, Dretler SP, K ahn RI, Lingeman JE, M acal uso J N J r, M cCullough DL. Nephrolithiasis Clinical Guidelines Panel Summery Report on The M anagement of Staghorn calculi: The A merican U rological A ssociation Nephrolithiasis Clinical Guidelines Panel. J U rol. 1994;151:1648-1651.

3. Stoller M L. U rinary Stone Disease. M cA ninch, JW \& L ue TF, (eds), Smith \& Tanagho's General U rology, 18 thedn. 2013;249-279. M cG rawHill, California.

4. Michel M S, Trojan L, Rassweiler JJ. Complications in Percutaneous N ephrolithotomy. EurU rol. 2007;51(4):899-906.

5. J ackman SV, Hedican, SP, Peters CA, D ocimo SG. Percutaneous nephrolithotomy in infants and pre-school age children: Experience with a new technique. World J U rol. 1998;52:697-701.

6. Chan DY, Jarrett TW. M ini-percutaneous nephrolithotomy. J Endourol. 2000;14:269-272.

7. Ferakis N, Stravroppoulos M. M ini percutaneous nephrolithotomy in the treatment of renal and upper ureteric stones: Lessons learned from a review of literature. U ro annals. 2015;7:141-148.

8. Li X, He Z, Wu K, Li SK, Zeng G, Yuan J, HeY, Lei M. Chinese minimally invasive percutaneous nephrolithotomy: The Guangzhou experience. J Endourol. 2009;23:693-697.

9. Wright A, Rukin N, Smith D, Rosette J, Somani BK. M ini, ultra, micro - nomenclature and cost of these new minimally invasive Percutaneous nephrolithotomy (PCNL) techniques. The A dvU rol. 2016;8(2):142-146.

10. Losty $P$, Surana $R, O^{\prime}$ D onnell B. Limitations of extracorporeal shock wave lithotripsy for urinary tract calculi in young children. J PedSurg. 1993;28:1037-1039.

11. J ackman SV, D ocimo SG, Cadeddu JA, B ishoff JT, K avoussi LR, J arrett TW. The "mini-perc" technique: A less invasive alternative to percutaneous nephrolithotomy. World J U rol. 1998;16:371-374.

12. Nagele U, A nastasiadis AG, Schilling DA, Sicrent KD, Kuczyk M A, Stenzl A. Introducing a new sealant applicator for easy, safe, and quick closure of a mini-percutaneous nephrolitholapaxy access tract. J Endourol. 2007;21:393-396.

13. J ackman SV, Hedican SP, D ocimo SG, Peters CA. M iniaturized access for pediatric percutaneous nephrolithotomy. J Endourol. 1997; 11: S133.

14. Helal M, Black T, Lockhart J, Figueroa TE. The Hickman peel-away sheath: A lternative for pediatric percutaneousnephrolithotomy. J Endourol. 1997; 11: 171-172.

15. Giusti G, Piccinelli A, Taverna G, Benetti A, Pasini L, Corinti M, Teppa A, Zandegiacomod ZS, Graziotti P. M iniperc? No, thank you! EurU rol. 2007;51:810-814.

16. Saxby M F, Sorahan T, Slaney P, Coppinger SW V. A case-control study of percutaneous nephrolithotomy versus extracorporeal shock wave lithotripsy. BrJ U rol. 1997;79: 317-323.

17. Lingeman JE, Coury TA, N ewman DM, Kahnoski RJ, M ertz JHO, M osbaugh PG, Steele RE, Woods JR. Comparison of results and morbidity of percutaneous nephrostolithotomy and extracorporeal shock wave lithotripsy. J U rol. 1987; 138:485-490.

18. Cheng $F, Y u W$, Zhang $X, Y$ ang $S, X i a ~ Y, R u a n Y$. M inimally invasive tract in percutaneous nephrolithotomy for renal stones. J Endouro. 2010;24:1579-1582.

19. Zhong W, Zeng G, Wu W, Chen W, Wu K. M inimally invasivepercutaneousnephrolithotomy with multiple mini tracts in a single session in treating Staghorncalculi.U rolRes;39:11722.

20. M ishraS, Sharma R,GargC, K urienA, SabnisR, Desai M. Prospective comparative study of minipercandstandard PNL for treatment of 1 to 2 cmsizerenal stone. BJUInt. 2011;108:8969.

21. A bdel hafez M F, A mend B, B edke J, K ruck S, Nagele U, Stenzl A, Schilling D. M inimally invasive percutaneous nephrolithotomy: A comparative study of The management of small and large renal stones. U rology. 2013;81:241-245.

22. Traxer O, Smith TG $3^{\text {rd }}$, Pearle MS, Corwin TS, Saboorian H, Cadeddu JA. Renal parenchymal injury after standard and mini percutaneous nephrostolithotomy. JU rol. 2001;165:1693-1695.

23. K noll T, Wezel F, M ichel MS, Honeck P, WendtN ordahl G. Do patients B enefit t from miniaturized tubeless percutaneous nephrolithotomy? A comparative prospective study. J Endourol. 2010;;24:1075-1079. 